\title{
Optimizing Multiscale SSIM for Compression via MLDS
}

\author{
Christophe Charrier, Member, IEEE, Kenneth Knoblauch, Laurence T. Maloney, \\ Alan C. Bovik, Fellow, IEEE, and Anush K. Moorthy
}

\begin{abstract}
A crucial step in the assessment of an image compression method is the evaluation of the perceived quality of the compressed images. Typically, researchers ask observers to rate perceived image quality directly and use these rating measures, averaged across observers and images, to assess how image quality degrades with increasing compression. These ratings in turn are used to calibrate and compare image quality assessment algorithms intended to predict human perception of image degradation. There are several drawbacks to using such omnibus measures. First, the interpretation of the rating scale is subjective and may differ from one observer to the next. Second, it is easy to overlook compression artifacts that are only present in particular kinds of images. In this paper, we use a recently developed method for assessing perceived image quality, maximum likelihood difference scaling (MLDS), and use it to assess the performance of a widely-used image quality assessment algorithm, multiscale structural similarity (MS-SSIM). MLDS allows us to quantify supra-threshold perceptual differences between pairs of images and to examine how perceived image quality, estimated through MLDS, changes as the compression rate is increased. We apply the method to a wide range of images and also analyze results for specific images. This approach circumvents the limitations inherent in the use of rating methods, and allows us also to evaluate MS-SSIM for different classes of visual image. We show how the data collected by MLDS allow us to recalibrate MS-SSIM to improve its performance.
\end{abstract}

Index Terms-Difference scaling, image quality assessment performance.

\section{INTRODUCTION}

$\mathbf{L}$ OSSY image compression techniques such as JPEG2000 allow high compression rates, but only at the cost of

Manuscript received December 3, 2010; revised June 20, 2012; accepted July 18, 2012. Date of publication July 30, 2012; date of current version November 14, 2012. This work was supported in part by the ANR Project ANR-08-SECU-007-04 and Intel and Cisco, Inc. under the VAWN Program. The associate editor coordinating the review of this manuscript and approving it for publication was Dr. Jesus Malo.

C. Charrier is with Université de Caen-Basse Normandie, GREYC Laboratory, Equipe Image, ENSICAEN, Caen 14050, France (e-mail: christophe.charrier@unicaen.fr).

K. Knoblauch is with the Département Neurosciences Intégratives, Institut National de la Santé et de la Recherche Médicale, Stem Cell and Brain Research Institute, Bron 69500, France (e-mail: ken.knoblauch@inserm.fr).

L. T. Maloney is with the Department of Psychology, Center for Neural Science, New York University, New York, NY 10012 USA (e-mail: 1 tm1@nyu.edu).

A. C. Bovik and A. K. Moorthy are with the Laboratory for Image and Video Engineering, University of Texas at Austin, Austin, TX 78712 USA (e-mail: bovik@ece.utexas.edu; anushmoorthy@gmail.com).

Color versions of one or more of the figures in this paper are available online at http://ieeexplore.ieee.org.

Digital Object Identifier 10.1109/TIP.2012.2210723 perceived degradation in image quality. There is a considerable literature concerning how human observers perceive compression-induced degradation in images and how well several Image Quality Assessment (IQA) algorithms tend to predict human judgments of reduction in image quality as a function of compression.

The most commonly employed means to assess human judgment of image quality is to ask human observers to rate image quality directly on a numerical scale. Human judgments are ordinarily expressed as the Mean Opinion Score (MOS) obtained from a sufficiently large set of human observer ratings relative to a normalized scale defined by the International Telecommunications Union (ITU) [1].

The typical summary of the agreement between rated subjective image quality and the ouput of an IQA algorithm is some measure of the correlation between the subjective ratings and the measured degree of distortion. Typical measures of correlation include 1) Pearson's linear correlation coefficient (CC) between MOS and algorithm score after nonlinear regression, 2) the root-mean-squared error (RMSE) between MOS and the algorithm score after nonlinear regression and 3) the Spearman rank order correlation coefficient (SROCC).

Examples of well-known IQA algorithms include DCtune [2], Picture Quality Scale (PQS) [3], Multi-Scale Structural SIMilarity (MS-SSIM) [4], Wavelet Structural Similarity (WSSI) [5], Visual Signal-to-Noise Ratio (VSNR) [6], and Visual Information Fidelity (VIF) [7] indices, to name a few. These indices compute relative quality scores between a reference image and a distorted version, often acheiving excellent correlations with MOS values. All those IQA indices have been designed using different frameworks. For example, MS-SSIM, WSSI and VIF were developed within a Natural Scene Statistics (NSS) framework or under assumptions about natural image structure. They are based on an assumption that distortion-free images occupy a small subspace of the space of all possible images. Image distortions can be interpreted as adding a distortion vector to distortion free images. DCtune and PQS were developed within a distortion-specific framework. They use distortion models based on a specific set of distortions (blockiness, blur, and so on) to predict quality scores. Any one of these algorithms can be judged better than a second if it correlates to a great extent with human MOS.

In [8], Sheik et al. compared 10 recent IQA algorithms and determined which had particularly high levels of performance. In [9], SESHADRINATHAN and BOVIK studied the relationship between the structural similarity and VIF frameworks and 


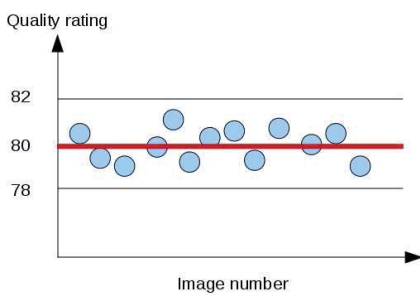

(a)

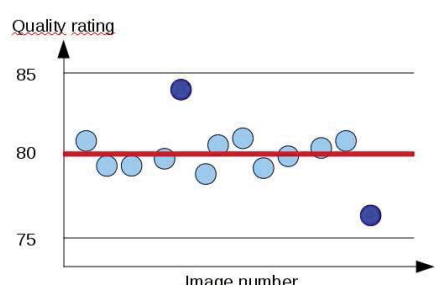

(b)
Fig. 1. Sample of the quality ratings obtained for each metric $q 1$ and $q 2$. (a) Distribution of the ratings obtained for the metric $q_{1}$. (b) Distribution of the ratings obtained for the metric $q_{2}$.

older metrics, i.e. the MSE and HVS-based quality metrics. They concluded that SSIM and VIF are closely related to the older IQA metrics under certain natural scene modeling assumptions. This was, also, recently studied by HoR $\tilde{A}$ and ZIOU who defined a bijective relation between SSIM and PSNR yielding predictions of SSIM values from PSNR (and inversely) [10]. The global conclusion of all those comparison studies is that no IQA algorithm has been shown to definitively outperform all others for all possible degradations, although owing to the inclusion of both scene models and perceptual models, the MS-SSIM and VIF indices tend to significantly outperform the others.

Moreover, typical evaluation measures can hide outliers (large, isolated failures). Consider two hypothetical IQA algorithms (say $q_{1}$ and $q_{2}$ ) that provide objective quality scores computed on a large database. Fig. 1(a) and 1(b) illustrate hypothetical samples of the obtained scores for each metric. For each subfigure, suppose the score equal to 80 represents the ground truth score and the grey circles are the computed scores for each image using $q_{1}$ (Fig. 1(a)) and $q_{2}$ (Fig. 1(b)). The samples are chosen so that the SROCC scores are identical for the two metrics (say 0.96) and this equality would typically be taken to mean that both IQA algorithms have the same global efficiency. Nevertheless, in Fig 1(a) and 1(b), one observes a difference in the distribution of the computed ratings. In Fig. 1(b), the distribution of ratings is very close to the ground truth rating except for two outliers. These two ratings are markedly in error. No such failure is visible in Fig. 1(a). If the isolated failures in $q_{2}$ are particularly costly we would conclude that the mediocre but dependable performance of IQA algorithm $q_{1}$ is preferable to the typically better but occasionally disastrous performance of $q_{2}$. Moreover, the occurrence of outliers may signal that the algorithm is ignoring some important aspect of human perception of images.

For example, when considering the MS-SSIM index [4], one can observe that despite a high degree of correlation with human ratings, it sometimes fails to accurately predict the quality score of a particular image. Fig 2 shows two such cases: 1) both human rating and predicted score of a degraded version of an original image are equivalent and equal to 71, and 2) human rating (54) and predicted score (27) of a degraded version of an original image are different.

Ultimately, however, the interpretation of human ratings and even a classification of outliers in human ratings is difficult. Suppose, for example, that the human observer rates two compressed (or otherwise distorted) images as 3 and 4 in

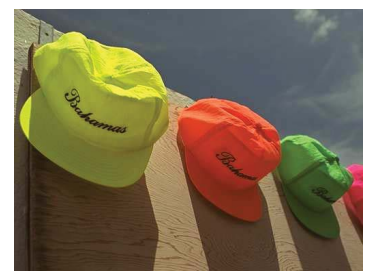

(a.i)

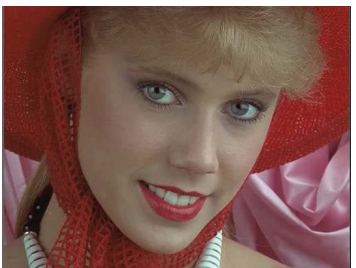

(b.i)

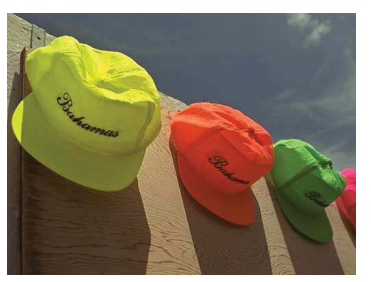

(a.ii)

(a)

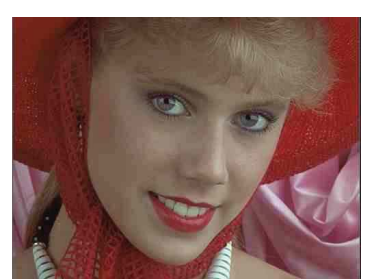

(b.ii) (b)

Fig. 2. Image extracted from the TID2008 image database (a) for which MSSSIM is in accordance with human rating and (b) for which MS-SSIM fails to accurately predict the human ratings. (a) Both human rating and predicted score of a degraded version (right) of an original image (left) are equivalent and equal to 71. (b) Human rating (54) and predicted score (27) of a degraded version (right) of an original image.

image quality (on say, a scale of 1 to 10) and also rates two other images as 7 and 8 , respectively. Although the difference in rating is the same for both pairs, we have no way to decide whether the perceived increase in quality between the first pair of images is equal to, greater than, or less than, the perceived increase in quality between the second pair. The subjective ratings only allow us to order the images by quality. Judgment of the importance of differences including assessment of the magnitudes of outliers is problematic.

CHARRIER et al. [11] recently applied a novel psychophysical method, Maximum Likelihood Difference Scaling (MLDS) [12]-[14] that circumvents this limitation of subjective rating methods. MLDS estimates an interval perceptual scale and, thus, makes it possible to quantify supra-threshold perceptual differences between pairs of images in order to evaluate perceptual changes in the images as compression-generated or other distortion is increased. The MLDS method is based on simple, forced-choice judgments and requires remarkably few trials to obtain quantitative estimates of the effects of any degree of distortion [11].

In this paper, we evaluate the efficacy of a recentlydeveloped general-purpose IQA algorithm in the specific context of compression-quality trade-off using MLDS. An investigation about its local variation to accurately predict the image quality score is performed, yielding a refinement of the IQA algorithm. The trial IQA algorithm that is used is the MS-SSIM index, due to its high degree of correlation with human ratings [4]. This paper is structured as follows. In Section II, we present the MLDS method. Section III summarizes MS-SSIM and its relevant parameters. In section IV, we discuss the evolution of the local correlation of the predicted ratings. The apparatus is also presented. In section $\mathrm{V}$, one approach to counterbalance the local lack of correlation is detailed and discussed. Section VI presents the results on two large public image quality assessment databases. This is followed by a concluding section. 


\section{Maximum Likelihood DifFerence ScAling}

Typical MOS algorithms are based on a psychophysical method introduced by Stevens in 1946 [15] known as magnitude estimation. In response to criticisms of the reliability of data collected using magnitude estimation, other scaling methods have been developed, among them the MLDS technique.

The MLDS method is based on forced-choice judgments of stimulus intervals and yields an interval scale of image degradation. The task underlying MLDS is not discrimination of images but direct comparison of suprathreshold differences between pairs of stimuli (images); the observer simply judges which of a pair of stimulus differences is greater. Avoiding the use of rating scales, the MLDS method avoids known problems associated with their use by human beings [16], [17].

MLDS has previously been used to estimate the effect of distortion level on perceived image quality [11]. Next we explain the model of the observer's judgments in the psychophysical task on which MLDS is based, using compression distortion as the application of interest.

An image series consists of a base image $\phi_{1}$ and compressed versions of the base image denoted $\phi_{2}, \ldots, \phi_{p}$, indexed by increasing degree of compression. If image $\phi_{i}$ is compressed to a greater degree than image $\phi_{j}$ we write $\phi_{i}>\phi_{j}$. For brevity, we denote images in the series by their subscripts. The pair $(i, j)$ will serve as shorthand for $\left(\phi_{i}, \phi_{j}\right)$.

On each trial, the observer views two pairs of stimuli $(i, j)$ and $(k, l)$ representing four different levels of compression of the initial image (including possibly no compression). We refer to these two pairs as a quadruple denoted $\{i, j ; k, l\}$. The observer judges whether the perceptual difference between the first pair $(i, j)$ is greater than that between the second pair $(k, l)$. Over the course of the experiment, the observer judges the differences of a subset of all possible quadruples (pairs of pairs) for the $N$ stimuli in the series $\phi_{1}, \ldots, \phi_{p}$. (i.e., $p$ compression levels).

The goal of MLDS is to assign numerical scale values $\left(\psi_{1}, \psi_{2}, \ldots, \psi_{p}\right)$ that can be used to predict how the observer orders the pairs in each quadruple. We refer to these values as a difference scale. In principle, we wish to assign these scale values so that the perceived difference between the images of the pair $(i, j)$ is judged greater than the perceived difference between the images of the pair $(k, l)$ if and only if,

$$
\left\|\psi_{i}-\psi_{j}\right\|>\left\|\psi_{k}-\psi_{l}\right\|
$$

The key advantage of a difference scale over rating data is that differences between scale values are readily interpretable since the judgments people make in MLDS are comparisons of differences. The magnitudes of outliers, in particular, are readily interpretable.

The full MLDS model and its fitting methods also allow for stochastic variation in human judgment. Intuitively, we expect such variation. If the differences $\left\|\psi_{i}-\psi_{j}\right\|$ and $\left\|\psi_{k}-\psi_{l}\right\|$ are close, it is unlikely that human observers would be so reliable in judgment as to always choose $(i, j)$ or always choose $(k, l)$ as predicted by the criterion (1). To take into account this judgment variation, MALONEY and YANG [12] proposed a model of difference judgment that allows the observer to exhibit stochastic variations in judgment. We next describe their model. Let $L_{i j}=\left\|\psi_{i}-\psi_{j}\right\|$ be the length of the interval $\left(a_{i}, a_{j}\right)$. The proposed decision model is an equal-variance, Gaussian, signal detection model [18], where the signal is the difference in the lengths of the intervals:

$$
\delta(i, j ; k, l)=L_{i j}-L_{k l}=\left\|\psi_{i}-\psi_{j}\right\|-\left\|\psi_{k}-\psi_{l}\right\|
$$

The signal $\delta$ is assumed to be contaminated by a Gaussian error $\epsilon$ with mean 0 and standard deviation $\sigma$ to form the judgment variable

$$
\Delta(i, j ; k, l)=\delta(i, j ; k, l)+\epsilon .
$$

MALONEY and YANG assumed that the observer, given the quadruple $(i, j ; k, l)$, selects the pair $(i, j)$ precisely when $\Delta(i, j ; k, l)>0$. The resulting model of the observer allows for stochastic variation in judgment. The observer, presented with the same stimuli, can give different responses. The degree of inconsistency predicted depends on the magnitude of $\delta(i, j ; k, l)$ relative to $\sigma$. This dependence can be used to test the model itself [12]-[14].

MALONEY and YANG [12] estimated the parameters of the MLDS model by the method of maximum likelihood using numerical optimization methods. KNOBLAUCH and MALONEY show how the scale values may also be estimated quickly and accurately using a Generalized Linear Model (GLM) [11], [13], [14]. They have integrated the functions necessary to perform these fits using either the direct or the GLM approach in an R package (MLDS) available from the Comprehensive R Archive Network (CRAN, accessible from http://www.rproject.org/). We used this package to estimate difference scales for each observer's data for each image. All scale estimations were carried out in the statistical language $\mathrm{R}$ using the mlds function.

If we add a constant $c$ to all the values on the difference scale $\left(\psi_{1}, \psi_{2}, \ldots, \psi_{p}\right)$ that maximizes likelihood, the resulting difference scale also maximizes likelihood. If we multiply all the values on the maximum likelihood difference scale $\left(\psi_{1}, \psi_{2}, \ldots, \psi_{p}\right)$ by a constant $a>0$, the resulting difference scale also maximizes likelihood once we scale $\sigma$ by $a$. Therefore, without loss of generality, we can fix the end points of the maximum likelihood difference scale to be $\psi_{1}=0$ and $\psi_{p}=1$. We report all our results in this normalized format.

\section{Test IQA Algorithm}

The MS-SSIM index [4] is a multiscale extension of the SSIM IQA algorithm introduced in [19]. MS-SSIM contains three factors pertaining to: 1) luminance distortion, 2) contrast distortion and 3) structure comparison.

All of these are first computed within multi-scale subband local patches and then pooled together to obtain the final predicted score between an original image and its degraded version.

The basis of this measure lies in the representation of an image as a vector within an image space. Any image distortion can be interpreted as adding a distortion vector to the reference image vector. In this space, the two vectors that 
represent luminance and contrast changes span a plane that is specific to the reference image vector. The image distortion corresponding to a rotation of such a plane by an angle can be interpreted as a structural change.

The luminance comparison is defined as

$$
l(I, J)=\frac{2 \mu_{I} \mu_{J}+C_{1}}{\mu_{I}^{2}+\mu_{J}^{2}+C_{1}}
$$

where $\mu_{I}$ and $\mu_{J}$ respectively represent the mean intensity of the image $I$ and $J$, and $C_{1}$ is a constant which prevents instability when $\mu_{I}^{2}+\mu_{J}^{2} \approx 0$. A common choice for the stabilizing constant is $C_{1}=\left(K_{1} L\right)^{2}$, where $L$ is the theoretical dynamic range of the image's pixels and $K_{1}=0.01$.

The contrast distortion measure is defined to have a similar form:

$$
c(I, J)=\frac{2 \sigma_{I} \sigma_{J}+C_{2}}{\sigma_{I}^{2}+\sigma_{J}^{2}+C_{2}}
$$

where $C_{2}$ is a non negative constant commonly defined as $C_{2}=\left(K_{2} L\right)^{2}\left(K_{2}=0.03\right)$, and $\sigma_{I}$ (resp. $\left.\sigma_{J}\right)$ represents the standard deviation.

The structure comparison is performed after luminance subtraction and contrast normalization. The structure comparison function is defined as:

$$
s(I, J)=\frac{\sigma_{I, J}+C_{3}}{\sigma_{I} \sigma_{J}+C_{3}}
$$

where $C_{3}$ is a non negative constant defined as $C_{3}=C_{2} / 2$, and $\sigma_{I, J}=\frac{1}{N-1} \sum_{i=1}^{N}\left(I_{i}-\mu_{I}\right)\left(J_{i}-\mu_{J}\right)$. Substituting $C_{3}$ by $C_{2} / 2$ in 6 :

$$
s(I, J)=\frac{2 \sigma_{I, J}+C_{2}}{2 \sigma_{I} \sigma_{J}+C_{2}}
$$

Note that $s(I, J)$ can be negative (e.g., if the subband is inverted). To obtain a multi-scale index, a blur/downsample operation is recursively applied on the reference $(I)$ and the distorted images $(J)$ to generate $M$ scales, as follows. The original scale is referred to as scale 1 , and the highest scale as scale $M$. Finally MS-SSIM is given by combining the luminance comparison (4), the contrast distortion measure (5) and the structure comparison (7) at different scales by:

$$
\operatorname{MS}-\operatorname{SSIM}(I, J)=\left[l_{M}(I, J)\right]^{\alpha_{M}} \prod_{i=1}^{M}\left[c_{i}(I, J)\right]^{\beta_{i}}\left[s_{i}(I, J)\right]^{\gamma_{i}}
$$

where the contrast comparison and the structure comparison are computed at the $i^{t h}$ scale, and denoted as $c_{i}(I, J)$ and $s_{i}(I, J)$, respectively; the luminance comparison $l_{M}(I, J)$ is computed only at scale $M$. The $2 M+1$ exponents $\alpha_{M}, \beta_{i}$ and $\gamma_{i}, i=1, \ldots, M$ are used to adjust the relative importances of the components. In the commonly used implementation [4], $M=5$ corresponds to the maximum scale, while $i=1$ corresponds to the original resolution of the image. In [4], the authors defined $\beta_{1}=\gamma_{1}=0.0448, \beta_{2}=\gamma_{2}=0.2856$, $\beta_{3}=\gamma_{3}=0.3001, \beta_{4}=\gamma_{4}=0.2363$, and $\alpha_{5}=\beta_{5}=\gamma_{5}=$ 0.1333 .

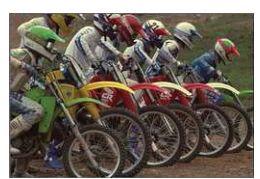

(a)

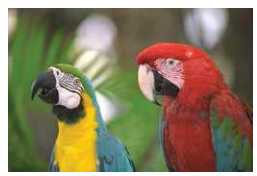

(d)

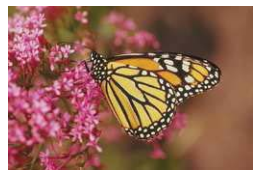

(g)

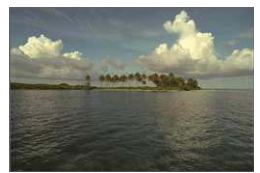

(j)

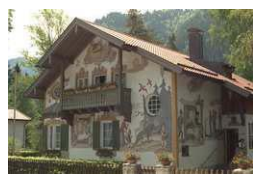

(m)

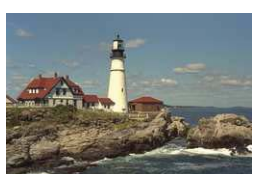

(b)

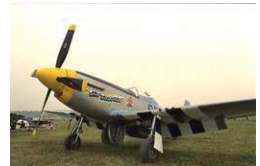

(e)

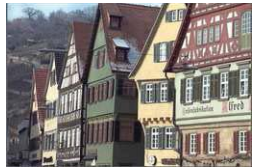

(h)

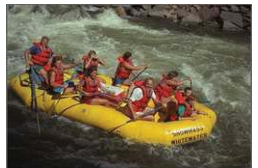

(k)

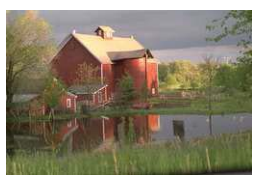

(n)

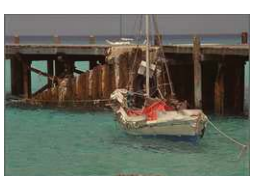

(c)

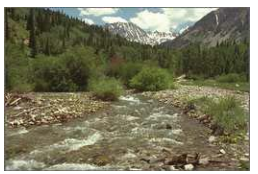

(f)

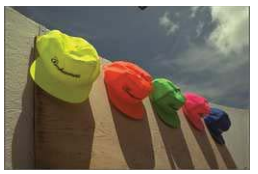

(i)

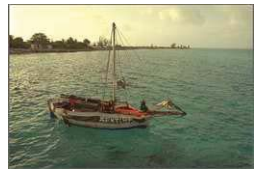

(1)

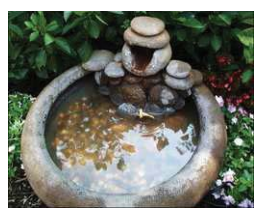

(o)
Fig. 3. 15 images used in the experiments are shown, with mnemonic labels. For each image, we estimated a difference scale based on each observer's judgments, yielding a total of 450 difference scales. (a) Imga, (b) imgb, (c) imgc, (d) imgd, (e) imge, (f) imgf, (g) imgg, (h) imgh, (i) imgi, (j) imgj, (k) imgk, (l) imgl, (m) imgm, (n) imgn, and (o) imgo.

\section{Evolution of the Correlation With Respect TO COMPRESSION RATE}

\section{A. Apparatus}

Thirty observers participated in the psychophysical tests. All observers had normal color vision (Ishihara test) and normal or corrected-to-normal acuity (Snellen test).

We computed 15 image series using the base images shown in Fig. 3. These images portray a variety of scenes and differ in their distributions of spatial and chromatic detail.

The size of images was typically $768 \times 512$ pixels or of similar size. For each visual test, the viewing distance was fixed at 32 pixels per degree of visual angle.

Before the main MLDS experiment we tested whether observers could correctly order the compressed images in descending order of quality. If they could not do so, even allowing for possible difficulty in discriminating adjacent images in the scale, there could be no difference scale that could account for their performance.

During this initial test, observers had to first select the highest quality image, then the second highest, etc. A sample trial is shown in Fig. 4. During the test, each time an image was selected by clicking on it, the selected image disappeared and the number of the rank order was shown. If the observer decided to cancel his choice, s/he just had 


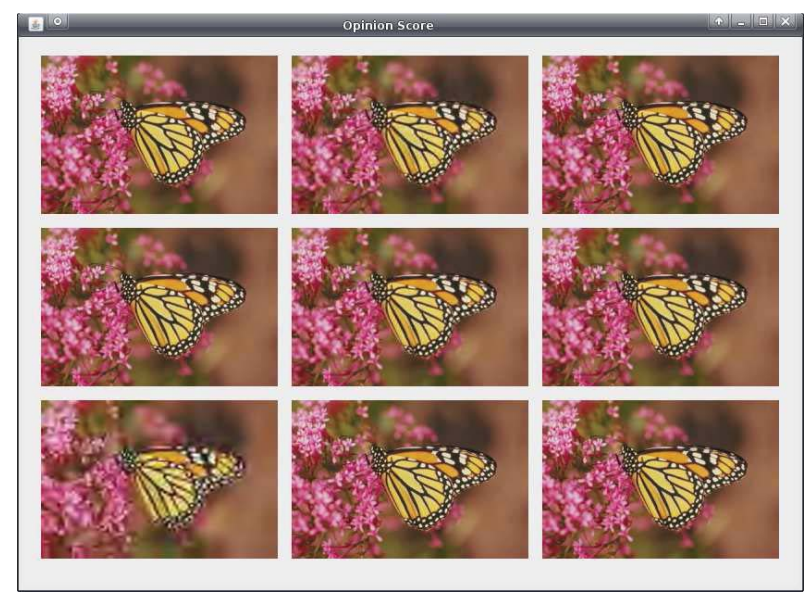

Fig. 4. Example of a single trial during the ordering test. The subject sees an image at the nine trial compression rates. The stimuli are randomly arranged on three lines. The subject was asked to order the quality of all images from the best to the worst quality.

to click on the rank order number. The corresponding image was shown again and the rank order number disappeared. In addition, s/he could deselect more than one image, depending on the selected number, for example, if the observer had already classified six images, the observer could deselect any image numbered from 1 to 6 . If s/he deselected image numbered 3, all images from 3 to 6 were automatically deselected.

During the main part of the experiment, the observer saw a quadruple of images drawn from a single image series. These four images were arranged as two pairs $(i, j)$ and $(k, l)$ on a computer display. On half of the trials, the first pair was displayed on the upper half of the display screen, the second on the lower, and on the remaining trials the first pair was displayed on the lower, the second on the upper. For the convenience of the observer, the less compressed of the two images in each pair was always on the left. The observer then judged which pair (upper or lower) exhibited the larger change or difference in quality. A sample trial is shown in Figure 5. Over the course of the experiment, the observer judged several hundred quadruples. These judgments were used to construct a numerical difference scale that captures the effect of additional compression on image quality [12], [13].

We applied MLDS to evaluate the image quality of the 15 trial original images, each compressed with JPEG2000 to nine different levels: $\{0.1000,0.3057,0.5627,0.7684,0.9741$, $1.1798,1.3854,1.5912\} \mathrm{bpp}$, plus the original image. We used the JPEG2000 implementation provided by The JasPer Project [20]. We obtained difference scales for each subject and image.

In order to compare MLDS values with scores obtained from the MS-SSIM IQA algorithm, we computed the score provided by the IQA algorithm for each of the nine trial images. Then the difference of scores for each pair of consecutive images was computed. Those differences were then cumulated across the series.

We then computed the correlations between cumulated MS-SSIM scores and MLDS values.

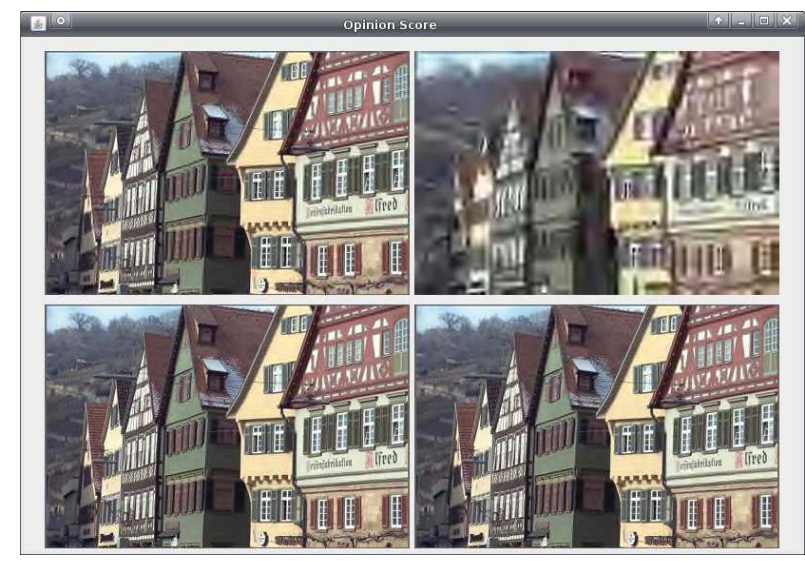

Fig. 5. Example of a single trial in MLDS. The subject was presented with an image at four different compression rates. The stimuli are arranged as two pairs $(i, j)$ and $(k, l)$. In each pair, the right-hand stimulus was more compressed. The subject was asked to judge whether the decrease in quality in going from $i$ to $j$ is greater than the decrease in going from $k$ to $l$. In this example, most observers would judge that the upper pair exhibits the larger change.
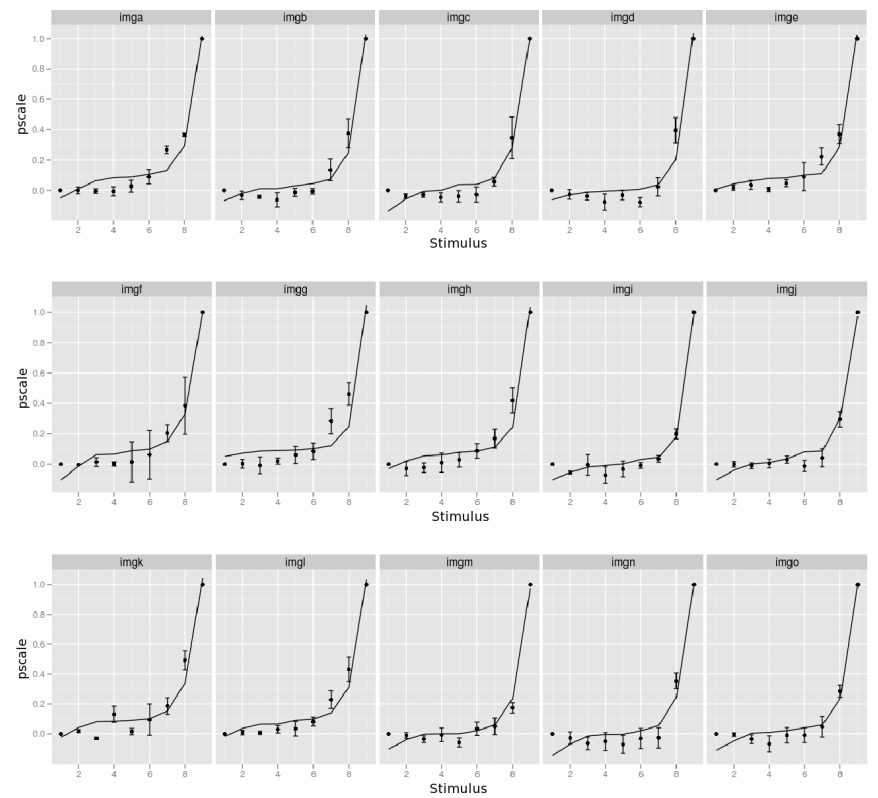

Fig. 6. Obtained results for all trial images and for the thirty observers. The black points and the black curve, respectively, represent the MLDS and the MS-SSIM values.

\section{B. Results}

The obtained results (Fig. 6) show that MS-SSIM captures perceptual changes in images with increasing compression rates very well. Yet, even if MS-SSIM globally yields high correlations with the judgment of human observers, sometimes it fails to accurately predict perceptual changes between images as the compression rate is increased. For example, considering the image imgk, observers have judged a high visible difference between stimulus 3 and 4 , whereas the associated MS-SSIM values are nearly identical.

In order to investigate these individual failures (outliers), the same procedure that was used to compare the scores obtained from the IQA algorithm and MLDS values was used for each one of the three factors embedded within MS-SSIM. 

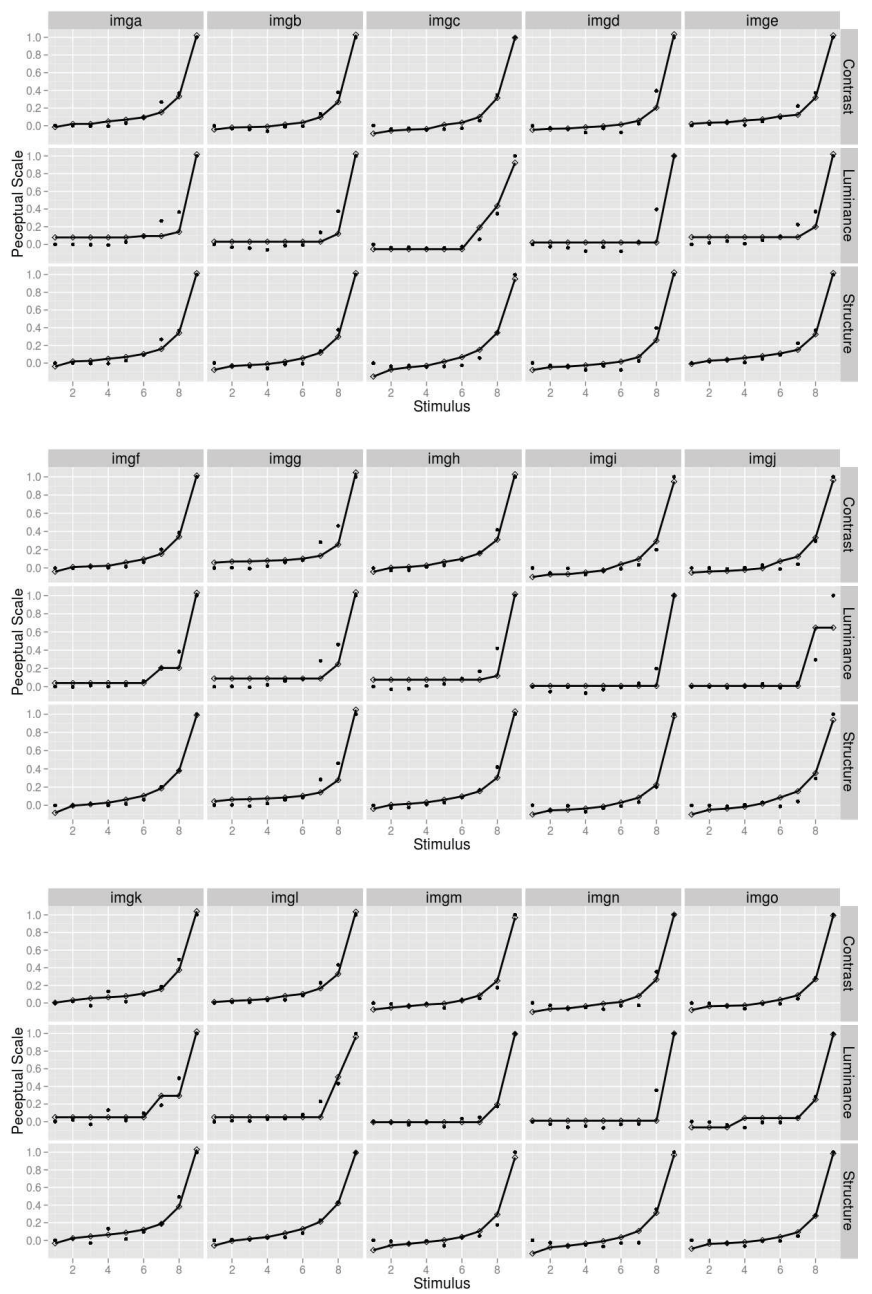

Fig. 7. Obtained results for all trial images. The black points and the curve, respectively, represent the MLDS and each of the three MS-SSIM factor values. For each of the three subfigures, the first row of each subimage corresponds to the contrast comparison values $\prod_{i=1}^{M} c_{i}(I, J)^{\beta_{i}}$, the second row corresponds to luminance comparison values $l_{M}(I, J)^{\alpha_{M}}$, and the last row represents the structure comparison values $\prod_{i=1}^{M} s_{i}(I, J)^{\gamma_{i}}$.

The results are shown in Fig. 7 for all trial images. The first row of each of the three subfigures corresponds to the contrast comparison values $\prod_{i=1}^{M} c_{i}(I, J)^{\beta_{i}}$, the second row corresponds to the luminance comparison values $l_{M}(I, J)^{\alpha_{M}}$, while the last row represents the structure comparison values $\prod_{i=1}^{M} s_{i}(I, J)^{\gamma_{i}}$. At first glance, one might remark that the third factor is less well correlated with MLDS than the two other factors, especially at the beginning of the scale. The same remark can be made when one compares the MLDS values to MS-SSIM in Fig. 6. A poor fit is observed at the beginning of most curves. Thus, structure comparison $\prod_{i=1}^{M} s_{i}(I, J)$ has great influence on the MS-SSIM values, as suggested in [21].

To achieve the best fit possible, one has to modify the influence of this third parameter. This can be done by changing the five $\gamma_{i}$ exponents. To perform this change, we first investigated the influence of the decomposition level $M$ on the fitting with MLDS values.

Since the third (structure) factor is initially computed using $M=5$ levels, we first investigated the influence of $M$ : how does $M$ influence the curve for this third factor? To measure

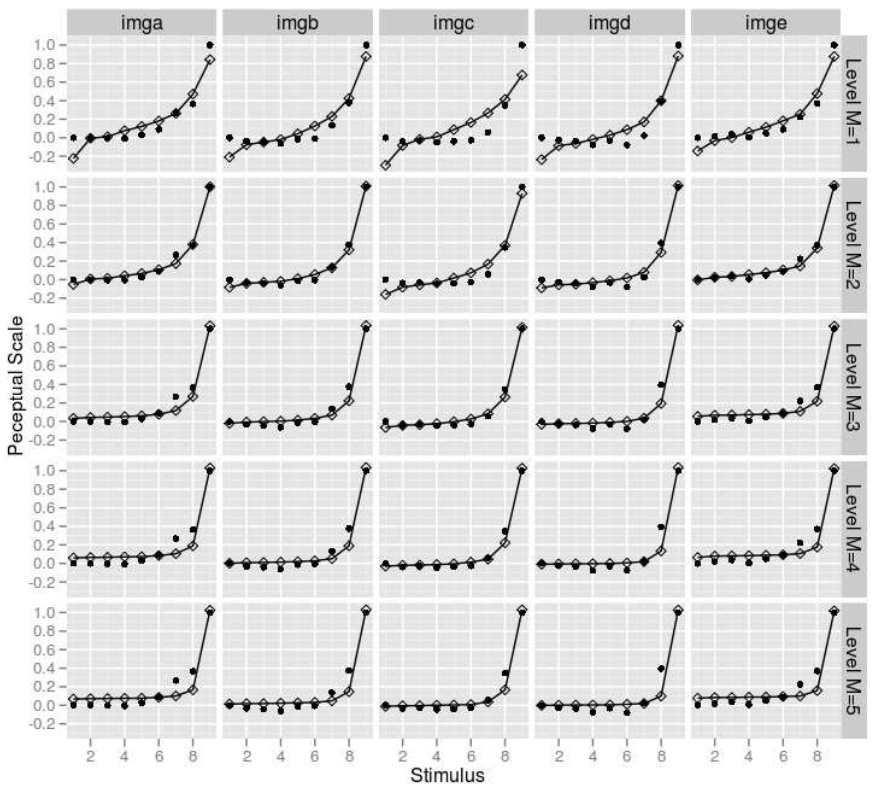

Fig. 8. Structure comparison feature values (7) as used to compute the MSSSIM values, for different decomposition levels and for the five first images (image to image). The black points and the black curve, respectively, represent the MLDS and the third factor values used to compute MS-SSIM. Each row corresponds to a decomposition level.

this influence, we computed the structure comparison factor for levels from 1 to 5. The obtained results are shown in Fig. 8 for a representative subset of trial images (imga to imge), where the black points and the black curve respectively represent the MLDS and the third factor values.

At each decomposition level, one can observe poor fits at the beginning of each scale, for each trial image. This poor fit is observed for low decomposition level values $(M=1$, $M=2$ ). The best fitting curve occurs at the third level, on average.

In order to reduce this lack of fit, we first investigated a basic weighting rule that consists of modifying the weight value on the third factor [22]. The main goal is to obtain a better fit of the third MS-SSIM values to MLDS. We found that refining the exponents values for the third MS-SSIM factor $s(.,$.$) reduced the failure observed at the beginning of the scale$ (Fig. 6), while the rest of the curve is unaffected, yielding a higher correlation value with human judgments.

From this, it can be presumed that to improve the correlation of the MS-SSIM IQA algorithm scores and MLDS, the coefficients $\left(\beta_{i}, \gamma_{i}\right)$ do not necessarily have to be identical (as initially suggested in [4]).

Furthermore, in MS-SSIM only luminance measured at the at the coarsest scale (fifth level) is used with exponent value equal $\alpha_{5}=\beta_{5}$. Note that $\alpha_{5}$ does not necessarily have to be equal to $\beta_{5}$ and allowing them to be unequal or making use of luminance information from other resolution levels may lead to a higher degree of correlation between MS-SSIM and human data.

Thus, next we investigate the impact of letting all of the parameters, $\alpha_{i}, \beta_{i}$ and $\gamma_{i}$, vary. Thus we will estimate 15 coefficient values to improve the MS-SSIM IQA algorithm. 


\section{IQA ALGORITHM GENETICALly IMPROVED}

Given the obtained results from the weight coefficient $\kappa$ for the third MS-SSIM factor, we hypothesize that different exponent values for each of the three attributes embedded in the MS-SSIM index would provide a higher global correlation rate.

\section{A. Associated Error Function}

The main objective is to find new exponent values for each decomposition scale of MS-SSIM. The associated formula can be expressed as a 15-parameter function:

$$
\begin{aligned}
\operatorname{MS}-\operatorname{SSIM}\left(I, J, \alpha_{i}, \beta_{i}, \gamma_{i} ; i=1, \ldots, M\right) \\
=\prod_{i=1}^{M}\left[l_{i}(I, J)^{\alpha_{i}} c_{i}(I, J)^{\beta_{i}} s_{i}(I, J)^{\gamma_{i}}\right]
\end{aligned}
$$

where $\sum_{i=1}^{M} \alpha_{i}+\beta_{i}+\gamma_{i}=1$ and $\forall i \in[1, \ldots, M], 0 \leq \alpha_{i} \leq$ $1,0 \leq \beta_{i} \leq 1,0 \leq \gamma_{i} \leq 1$.

From (9), the search for the new exponent values seeks minimization of the error function

$$
\begin{aligned}
& E\left(\alpha_{i}, \beta_{i}, \gamma_{i} ; i=1, \ldots, M\right) \\
& =\min \left(\sum_{j=1}^{K}\left(\operatorname{MLDS}_{j}(I, J)-\mathrm{fMS}^{-\operatorname{SSIM}_{j}}\left(I, J, \alpha_{i}, \beta_{i}, \gamma_{i}\right)\right)^{2}\right)
\end{aligned}
$$

where $K$ is the number of tested images for which the MLDS values are provided, and $\mathrm{fMS}_{-\mathrm{SSIM}_{j}}($.$) are the computed rates$ obtained following a logistic regression.

In other words, the goal is to estimate the 15 exponent values that minimize the error function $E($.$) . Since the error func-$ tion is non-convex and may contain numerous local optima, the choice of search strategy to optimize it is important.

\section{B. Search Strategy}

In this section, the problem of defining a suitable search strategy is addressed. The retrieval of the minimum between the MLDS value and the MS-SSIM value is a global optimization problem, where the error function $E($.$) is minimized with$ respect to a set of parameters as in (9). More specifically, the error function (Eq. 10) defines a non-linear multidimensional function, usually characterized by several local minima. Therefore, the search strategy should find the global minimum, and avoid remaining trapped in local minima. Two problems must be successfully treated 1) the large search space and 2) false matches corresponding to local minima.

The simplest way to find $\left(\alpha_{i}, \beta_{i}, \gamma_{i}\right)_{i \in[1, \ldots, M]}$ is by considering a large number of $\left(\alpha_{i}, \beta_{i}, \gamma_{i}\right)_{i \in[1, \ldots, M]}$ values, keeping the one whose MS-SSIM value is the closest to MLDS (i.e. the one with the lowest error $E()$.$) . Of course, the more samples$ considered, the more precise the end result will be. This kind of brute-force approach based on searching all possible combinations of parameters is not feasible in practice.

The Genetic Algorithm (GA) is a population-based stochastic search procedure that finds exact or approximate solutions to optimization and search problems. Modeled on the mechanisms of evolution and natural genetics, genetic algorithms provide an alternative to traditional optimization techniques by using directed random searches to locate optimal solutions in multimodal landscapes [23]. Their basic principles were first introduced by Holland in 1975 [24] and extended to functional optimization by De Jong [25] and Goldberg [26], and have since proven to be efficient and stable in searching for global optimum solutions [27]-[29]. One of the most attractive features of GAs is their ability to solve problems involving non-differentiable functions and those defined in discrete as well as continuous spaces.

Usually, a simple GA is composed of three operations: selection, genetic operation, and replacement. GAs use a population, which is composed of a group of chromosomes, to represent the solutions of the system. Defining the solution representation of the system is the first task when applying GAs. The solution in the problem domain can then be encoded into the chromosome in the GA domain, and vice versa. Initially, a population is randomly generated. The fitting function then uses values from objective functions to evaluate the quality of fit of each chromosome.

The "fitter" chromosome has the greater chance to survive during the evolution process. The objective function is problem specific; its objective value can represent the system performance index (e.g., an error). Next, a particular group of chromosomes is chosen from the population to be parents. The offspring are then generated from these parents using genetic operations, which normally are crossover and mutation. Similar to their parents, the fitness of the offspring are evaluated and used in replacement processes in order to replace the chromosomes in the current population by the selected off-spring. The GA cycle is then repeated until a desired termination criterion is satisfied, for example, the maximum number of generations is reached, or the objective value is below the threshold.

In this paper, $M=5$ is the number of levels used to compute the MS-SSIM value. In this case, the GA domain represents a 15-dimensional space in which one point is expressed as $\left(\alpha_{1}, \ldots, \alpha_{M}, \beta_{1}, \ldots, \beta_{M}, \gamma_{1}, \ldots, \gamma_{M}\right)$, and the fitness function is defined by (10).

\section{Optimization Results}

To seek each exponent value, the 15 reference images JP2K compressed at nine different compression level as depicted in section IV-A are used to compute the 15 multilevel features $l_{i}(I, J), c_{i}(I, J)$ and $s_{i}(I, J) \forall i \in[1, \ldots, 5]$.

Table I shows the estimated values for each exponent after minimizing (10). Fig. 9 shows the comparison of the MLDS scale values and the 15 parameter fitted MS-SSIM model. The black points represents the MLDS values, the black continuous curves the MS-SSIM indices computed using the original exponent values and the dashed curves by computing MS-SSIM values with the exponent values from Table I. For each trial image (imga to imgo), a better fit to the MLDS values was obtained when the MS-SSIM values are computed with the new exponent values than with the original ones. 
TABLE I

15 Computed Exponents And Associated Confidence Intervals (CI) With a 95\% Confidence Level Using A GA Approach Under the Constraints $\sum_{i=1}^{M} \alpha_{i}+\beta_{i}+\gamma_{i}=1$ And $\forall i \in[1, \ldots, M], 0 \leq \alpha_{i} \leq 1,0 \leq \beta_{i} \leq 1,0 \leq \gamma_{i} \leq 1$

\begin{tabular}{|r||c|c|c|c|c|}
\hline \hline Exponent & $\alpha_{1}$ & $\alpha_{2}$ & $\alpha_{3}$ & $\alpha_{4}$ & $\alpha_{5}$ \\
\hline Value & 0.1920 & 0.2169 & 0.2026 & 0.2136 & 0.1749 \\
$\mathrm{CI}$ & {$[0.0989,0.2415]$} & {$[0.1877,0.2791]$} & {$[0.1692,0.2384]$} & {$[0.1765,0.2868]$} & {$[0.0814,0.2304]$} \\
\hline \hline Exponent & $\beta_{1}$ & $\beta_{2}$ & $\beta_{3}$ & $\beta_{4}$ & $\beta_{5}$ \\
\hline Value & 0.9612 & 0.0097 & 0.0097 & 0.0097 & 0.0097 \\
$\mathrm{CI}$ & {$[0.8288,0.9681]$} & {$[-0.0145,0.0933]$} & {$[0.0084,0.0112]$} & {$[0.0084,0.0112]$} & {$[-0.0133,0.1012]$} \\
\hline \hline Exponent & $\gamma_{1}$ & $\gamma_{2}$ & $\gamma_{3}$ & $\gamma_{4}$ & $\gamma_{5}$ \\
\hline Value & 0.0082 & 0.1586 & 0.8167 & 0.0083 & 0.0082 \\
CI & {$[0.0073,0.0086]$} & {$[0.1241,0.2530]$} & {$[0.7250,0.8501]$} & {$[0.0073,0.0086]$} & {$[0.0073,0.0086]$} \\
\hline \hline
\end{tabular}
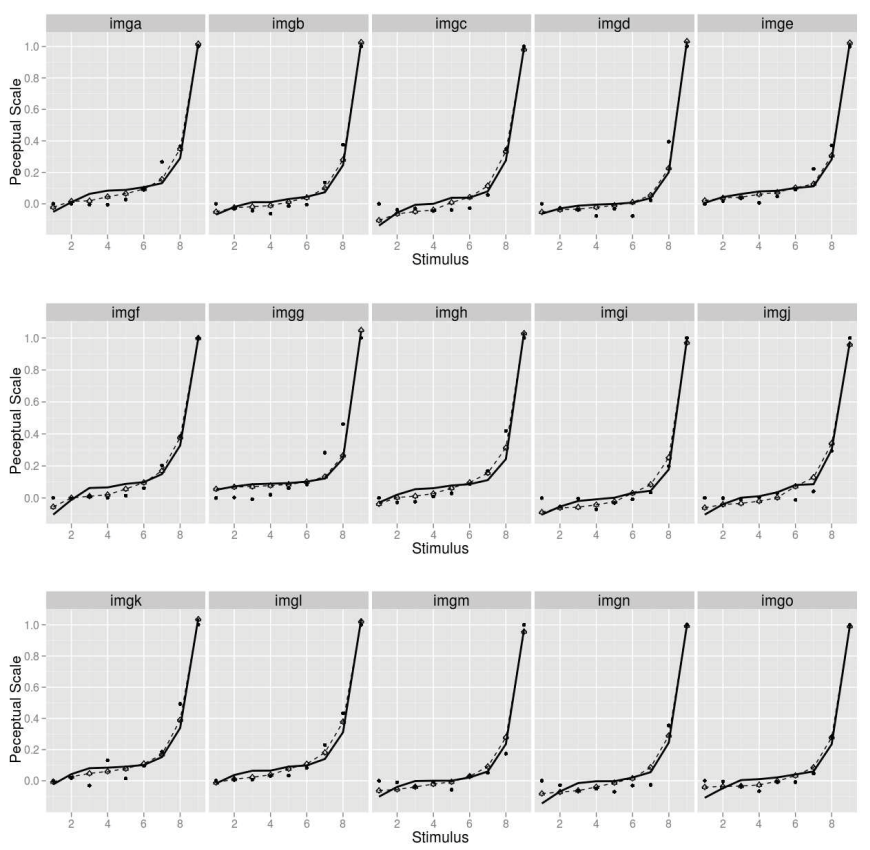

Fig. 9. Obtained results for all trial images. The black points represent the MLDS values, the black curve is associated with the original MS-SSIM index, and the dashed curve represents the computed MS-SSIM values using the new exponent values.

Table II presents the MSE obtained using the original exponent values and the new ones for all trial images. A reduction of more than 0.2 was attained. This means that the new MS-SSIM indices are better correlated to the MLDS values than the original ones. This is not really surprising, since minimizing the error function $E($.$) is the basis for deriving those new$ values. In addition, confidence intervals with a $95 \%$ confidence level are provided for each exponent. They are computed using a boostrap process with 999 replicates.

In order to take into account local correlation to design an IQA algorithm, one can define and minimize an error function between MLDS values and the predicted values. This can help improve the design of the test IQA method, i.e. the MS-SSIM measure by finding new exponent values. Fig. 10 displays the original exponent values (black points) and the new ones (black stars) for the three multiscale parameters embedded in MS-SSIM. If we consider the associated coefficients
TABLE II

COMPUTED MSE FOR BOTH ORIGINAL MS-SSIM INDEX AND FOR MS-SSIM INDEX USING NEW EXPONENTS USING A LINEAR REGRESSION WITH RESPECT TO MLDS VALUES

\begin{tabular}{|r|c|c|}
\hline \hline MS-SSIM & Original weighted & New weighted \\
\hline MSE & 0.6092 & 0.3863 \\
\hline
\end{tabular}

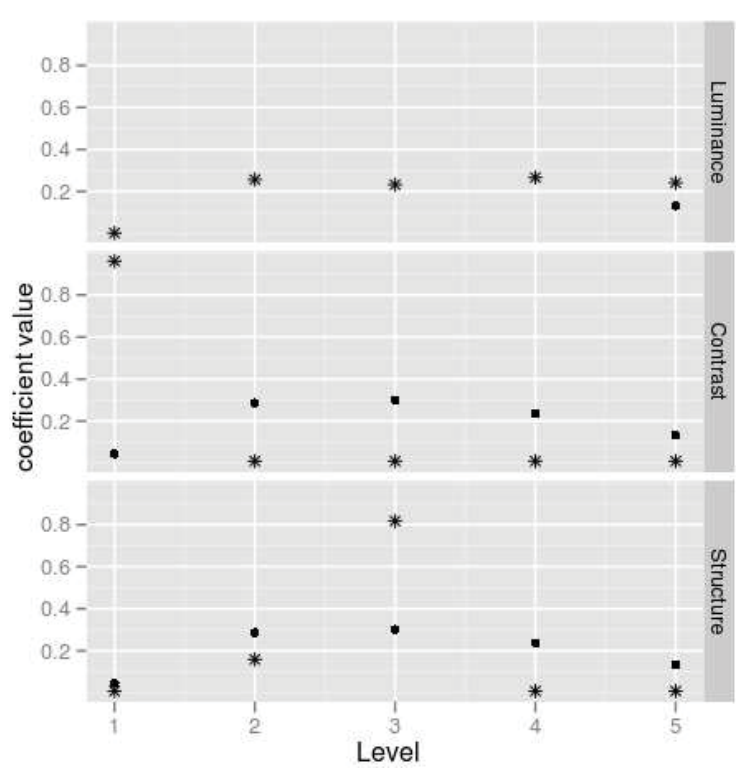

Fig. 10. Original exponent values (black points) and the new ones (black stars) for each MS-SSIM attribute.

for the structure attribute (third line), we observe that the third decomposition level seems to be considered of greater importance since its exponent value is higher whereas the four others are quite similar. Analyzing Fig. 8, one can see that this level is the best in terms of the fit to the MLDS values. The four other levels are quite similar in terms of fit with the MLDS values. The curve associated with these exponent values is quite similar to the curve associated with the original exponents. Nevertheless, considering individual correlation, the new exponent value associated to the third level is of higher degree than the original one. This is mainly due to the fact that the structure attribute at the third level is a good estimator of the structure degradation evolution. 


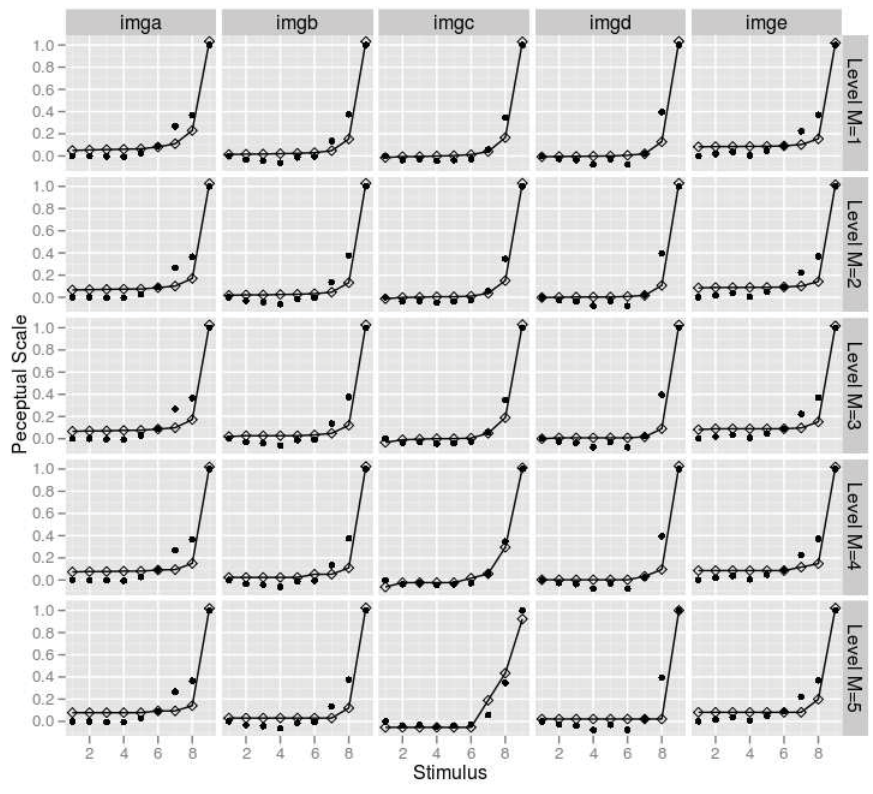

Fig. 11. Luminance feature values for the five decomposition levels and for the five first images (imga to imge). The black points and the black curve, respectively, represent the MLDS and the luminance factor values used to compute MS-SSIM. The $i$ th row corresponds to a $i$ th decomposition level.

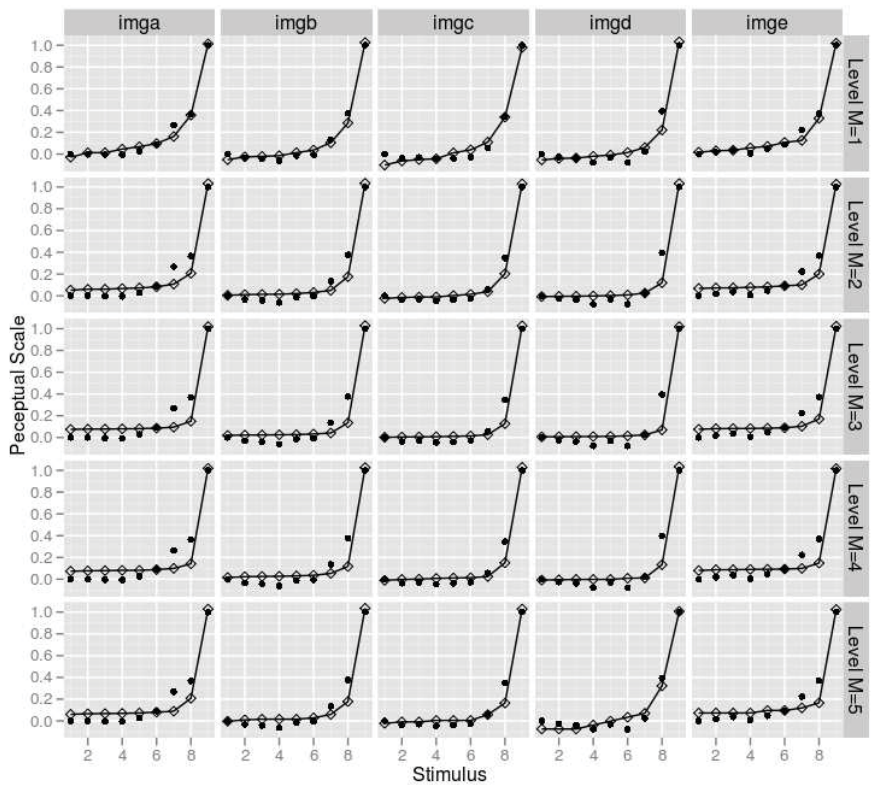

Fig. 12. Contrast feature values for the five decomposition levels and for the five first images (image through image). The black points and the black curve, respectively, represent the MLDS and the third factor values used to compute MS-SSIM. The $i$ th row corresponds to a $i$ th decomposition level.

If we consider the multi-level luminance attribute, only the fifth level was originally considered to be of interest, since only $\alpha_{5}$ is used. But, if we observe Fig. 11 the fiting at each level is nearly identical. This suggests that all the five levels should contribute approximately equally to measurement of the luminance degradation.

Focusing on the contrast attribute (see Fig. 12), note that the first level fits the MLDS values quite well. For levels from 2 to 5 , one observes that the values are quite similar with lower
TABLE III

COMPUTED CORRELATION COEFICIENTS FOR BOTH ORIGINAL MS-SSIM INDEX AND FOR MS-SSIM INDEX USING NEW EXPONENTS USING A LINEAR REGRESSION WITH RESPECT TO DMOS VALUES FROM THE LIVE DATABASE

\begin{tabular}{|c|c|c|c|c|c|c|}
\hline \hline & \multicolumn{2}{|c|}{ JP2K } & \multicolumn{2}{c|}{ JPEG } & \multicolumn{2}{c|}{ White noise } \\
\hline & Original & New & Original & New & Original & New \\
\hline CC & 0.783 & 0.810 & 0.730 & 0.742 & 0.9153 & 0.9142 \\
\hline KROCC & 0.884 & 0.884 & 0.849 & 0.852 & 0.8887 & 0.8878 \\
\hline SROCC & 0.980 & 0.991 & 0.962 & 0.981 & 0.9825 & 0.9813 \\
\hline \hline & \multicolumn{2}{|c|}{ Gaussian blur } & \multicolumn{2}{c|}{ FastFading } & \multicolumn{2}{c|}{ All } \\
\hline & Original & New & Original & New & Original & New \\
\hline CC & 0.8864 & 0.8623 & 0.725 & 0.788 & 0.7980 & 0.8142 \\
\hline KROCC & 0.8591 & 0.8413 & 0.859 & 0.876 & 0.8021 & 0.8543 \\
\hline SROCC & 0.9725 & 0.9627 & 0.965 & 0.974 & 0.9464 & 0.9762 \\
\hline \hline
\end{tabular}

fitting accuracy. This agrees with the displayed values in Fig. 10 where the first value is higher than the others and the four last values are identical.

Following the above procedure, better local correlation is obtained, and thus, the error between the MLDS values and the predicted MS-SSIM indices is minimized. This implies that the refined MS-SSIM indices are better correlated to human judgments.

\section{Vi. Evaluation of the Performance of the Refined MS-SSIM INDEX}

In order to judge the impact of the 15 new exponents estimated in the previous section, we tested the refined MS-SSIM index on both the LIVE and the TID2008 Image Quality databases.

To provide quantitative performance evaluation, three measures of correlation have been used: 1) Pearson, 2) Kendall and 3) Spearman measures. To perform the Pearson correlation measures, a logistic function (as adopted in the video quality experts group (VQEG) Phase I FR-TV test [30]) was used to provide a non-linear mapping between the refined MSSSIM values and subjective scores. We then separately used the subjective scores provided with the overall LIVE and the TID2008 database. Kendall and Spearman correlation measures were computed between the DMOS values and the MS-SSIM indices obtained using both the original exponent values and the new ones (Table I). Those measures can be interpreted as prediction accuracy measures (Pearson and Kendall coefficients) and prediction monotonicity measure (Spearman coefficient).

\section{A. Results on the LIVE Database}

Considering first the LIVE database, the results are presented in Table III. The scatter plots of DMOS versus both the original and the refined MS-SSIM values are shown in Fig. 13, where each point represents one test image, the vertical and horizontal axes representing MOS and the given distortion objective quality score for the original MS-SSIM (black points) and the refined MS-SSIM values (crosses), respectively. 

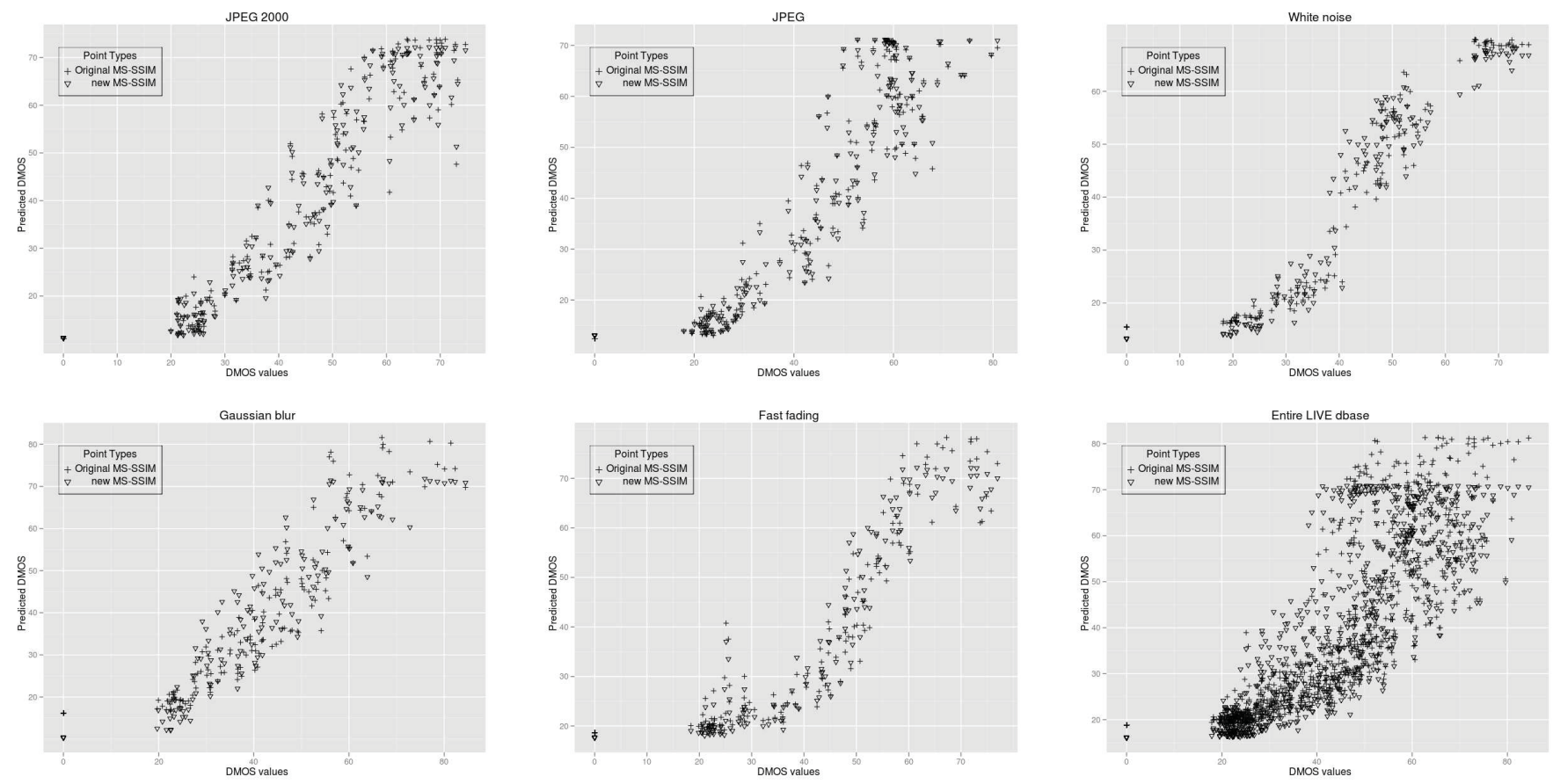

Fig. 13. Scatter plots of DMOS versus the original and MLDS-refined MS-SSIM predictions (with original exponent values and the new ones). Each point represents one test image in the LIVE image database.

TABLE IV

COMPUTED CORRELATION COEFFICIENTS FOR BOTH ORIGINAL MS-SSIM INDEX AND FOR MS-SSIM INDEX USING NEW EXPONENTS USING A Linear Regression With Respect to DMOS Values From the TID2008 Database. The Type of Degradations ARE Explained in TABLe V

\begin{tabular}{|c|c|c|c|c|c|c|c|c|c|c|c|c|}
\hline & \multicolumn{2}{|c|}{ Degrad \#1 } & \multicolumn{2}{|c|}{ Degrad \#2 } & \multicolumn{2}{|c|}{ Degrad \#3 } & \multicolumn{2}{|c|}{ Degrad \#4 } & \multicolumn{2}{|c|}{ Degrad \#5 } & \multicolumn{2}{|c|}{ Degrad \#6 } \\
\hline & Original & New & Original & New & Original & New & Original & New & Original & New & Original & New \\
\hline $\mathrm{CC}$ & 0.7994 & 0.7700 & 0.8151 & 0.7913 & 0.8278 & 0.8340 & 0.8341 & 0.8224 & 0.8861 & 0.8333 & 0.6672 & 0.6399 \\
\hline KROCC & 0.6139 & 0.5767 & 0.6013 & 0.5677 & 0.6148 & 0.6241 & 0.6117 & 0.5977 & 0.6419 & 0.5887 & 0.4846 & 0.4575 \\
\hline SROCC & 0.8099 & 0.7767 & 0.8055 & 0.7748 & 0.8215 & 0.8265 & 0.8099 & 0.7923 & 0.8706 & 0.8211 & 0.6899 & 0.6547 \\
\hline & \multicolumn{2}{|c|}{ Degrad \#7 } & \multicolumn{2}{|c|}{ Degrad \#8 } & \multicolumn{2}{|c|}{ Degrad \#9 } & \multicolumn{2}{|c|}{ Degrad \#10 } & \multicolumn{2}{|c|}{ Degrad \#11 } & \multicolumn{2}{|c|}{ Degrad \#12 } \\
\hline $\mathrm{CC}$ & 0.8524 & 0.8355 & 0.9384 & 0.9292 & 0.9638 & 0.9485 & 0.9629 & 0.9796 & 0.9727 & 0.9823 & 0.8784 & 0.8983 \\
\hline KROCC & 0.6569 & 0.6514 & 0.8169 & 0.7793 & 0.8316 & 0.8013 & 0.7489 & 0.7664 & 0.8559 & 0.8876 & 0.6637 & 0.6891 \\
\hline SROCC & 0.8488 & 0.8361 & 0.9563 & 0.9355 & 0.9587 & 0.9458 & 0.9328 & 0.9571 & 0.9697 & 0.9812 & 0.8663 & 0.8852 \\
\hline & \multicolumn{2}{|c|}{ Degrad \#13 } & \multicolumn{2}{|c|}{ Degrad \#14 } & \multicolumn{2}{|c|}{ Degrad \#15 } & \multicolumn{2}{|c|}{ Degrad \#16 } & \multicolumn{2}{|c|}{ Degrad \#17 } & \multicolumn{2}{|c|}{ All } \\
\hline $\mathrm{CC}$ & 0.8414 & 0.8437 & 0.7417 & 0.7388 & 0.7290 & 0.8666 & 0.7322 & 0.7259 & 0.7721 & 0.5468 & 0.8332 & 0.8532 \\
\hline KROCC & 0.6766 & 0.6957 & 0.5254 & 0.5335 & 0.5038 & 0.6309 & 0.5345 & 0.5427 & 0.4748 & 0.4068 & 0.6577 & 0.6699 \\
\hline SROCC & 0.8609 & 0.8849 & 0.7375 & 0.7434 & 0.7109 & 0.8353 & 0.7239 & 0.7402 & 0.6349 & 0.5430 & 0.8543 & 0.8601 \\
\hline
\end{tabular}

From both the scatter plots and the correlation evaluation results, we see that the performance of the MS-SSIM index computed with the new exponent values yields improved performance relative to the MS-SSIM values obtained with the original exponent values. This is not true for noisy or blurred images, since a decrease of the correlation coefficients is observed. Nevertheless, when all degradations are included, one observes that the SROCC is significantly higher when new exponent values are used. Naturally, this is driven in part by optimization of QA with respect to JP2K and also FastFading (which uses JP2K), but also JPEG distortion.

\section{B. Results on the TID Database}

Table IV displays the correlations obtained for both original MS-SSIM index and refined MS-SSIM index with respect to DMOS values from the TID2008 database. When the correlations relative to the subjective values were calculated on the TID2008 database, the refined MS-SSIM index again outperformed the originally designed MS-SSIM. If we only investigate compression artifacts (degradation \#10 to \#13, as defined in Table V), the refined MS-SSIM yields a significant increase of the correlation values. Similar to the results obtained from the LIVE image database, a decrease of the correlation values is globally observed for noise artifacts (degradation \#1 to \#7 and \#14). One notes that for two particular noise artifacts, an increase of the correlation value occurs (\#3 and \#14). A small increase of the correlation values is also obtained for degradation \#15 and \#16. Both non eccentricity pattern noise (\#14) and local block-wise distortions of different intensity (\#15) artifacts can be interprated as a block degradation of the image that is a typical compression artifact. This can explain 
TABLE V

DESCRIPTION OF THE 17 DEGRADATION TYPES Within THE TID2008 DATABASE

\begin{tabular}{|c|l|}
\hline Degrad no. & Type of distortion \\
\hline 1 & Additive Gaussian noise \\
2 & Additive noise in color components is more intensive \\
than additive noise in the luminance component \\
3 & Spatially correlated noise \\
4 & Masked noise \\
5 & High frequency noise \\
6 & Impulse noise \\
7 & Quantization noise \\
8 & Gaussian blur \\
9 & Image denoising \\
10 & JPEG compression \\
11 & JPEG2000 compression \\
12 & JPEG transmission errors \\
13 & JPEG2000 transmission errors \\
14 & Non eccentricity pattern noise \\
15 & Local block-wise distortions of different intensity \\
16 & Mean shift (intensity shift) \\
17 & Contrast change \\
\hline
\end{tabular}

the associated notable increase of the correlation, since the refined MS-SSIM index has been optimized for compression artifacts.

Degradations \#3 and \#16, respectively, concern a spatially correlated noise and a change of intensity. When analysing the images corresponding to degradation \#16, visible differences between the reference image and the degraded versions are not necessarily great. This could correspond to the first part of the obtained curves when the fitting with MLDS values is generated. Actually, from Fig. 9, a flat part is noticeable at the beginning of each curve. The refined MS-SSIM index seems to fit better this particular part than the original MS-SSIM. For JP2K compression artifacts, this particular part corresponds to slightly compressed images, where visible differences are not easily observable (that is the case for degraded images with artifact \#16). This can explain why higher correlation values are obtained for degradation \#16.

\section{Statistical Significance}

To assess whether the difference in performance between the original MS-SSIM index and the refined MS-SSIM index is statistically significant, we applied a variance-based hypothesis test using the residuals between the DMOS values and the ratings provided by the trial IQA algorithms. This test is based on the F-test that determines whether two population variances are equal. This is done by comparing the ratio of the two computed variances. The null hypothesis is that the residuals from the original MS-SSIM index are statistically indistinguishable (at a 95\% confidence level) from the residuals of the refined MS-SSIM. As mentioned in [8], the threshold ratio value for which the two sets of residuals are statistically distinguishable can be obtained from the F-distribution [31].

The results obtained from this test confirm that the difference of correlation over the entire LIVE database (Table III) is statistically significant at the 0.05 level.

Regarding the TID2008 database (Table IV), we found that the difference of correlation is not statistically significant overall, which is not surprising given the breadth of distortions in the TID database. However, we did find that the refined MS-SSIM index is superior to the original MS-SSIM index with statistical significance for degradations \#10 to \#13 and \#15. Those degradations concern artifacts that occur during a compression scheme applied on images.

\section{CONCLUSION}

When one judges the performance of IQA algorithms, correlations with human ratings are typically computed. The higher the correlation value is, the better the prediction score is. Absolute rating quality methods are usually used to obtain human ratings that will serve as ground truth (MOS or DMOS). Yet, image quality ratings (based on absolute judgments) are considerably less reliable than difference judgements, for reasons described in section II. Intead of using MOS (or DMOS) values which are obtained from quality ratings based on absolute judgments, we have used a recent psychophysical method, Maximum Likelihood Difference Scaling (MLDS) to evaluate IQA methods and improve them.

We applied it to a large collection of images to assess the consequences of JP2K compression and compared observers' judgments image quality to the predictions of one IQA method, MS-SSIM. We found that MS-SSIM suffers from local failures when assessing JP2K compression, especially due to its third (structure) factor that greatly influences the predicted values. It was found these local failures can be reduced using different values for the three $\left(\alpha_{i}, \beta_{i}, \gamma_{i}\right)$ exponents which we estimate from data. The refined MS-SSIM index was found to yield significantly improved performance relative to the original algorithm on two large public image quality assessment databases.

The use of MLDS permits interpretation of the correlation value of IQA algorithms across the series of degradations. This help us identify levels of degradation for which IQA can fail. This is not easily done when absolute rating quality methods are used instead of MLDS. This yields a more precise comparison to human ratings, and helps in the design of high performance IQA algorithms. This allowed us to improve the performance of MS-SSIM for compression-based distortions. Even if the results could be attributed to the use of JP2K compressed images to reweight MS-SSIM, the obtained overall performance for both LIVE and TID2008 database is better than using original MS-SSIM.

\section{REFERENCES}

[1] "Méthodologie d'évaluation subjective de la qualité des images de télévision,” ITU, Geneva, Switzerland, Tech. Rep. ITU-R BT.500-11, 2002.

[2] A. B. Watson, "DCTune: A technique for visual optimization of DCT quantization matrices for individual images," in Soc. Inf. Display Dig. Tech. Papers, vol. 24. 1993, p. 946-949. 
[3] M. Miyahara, K. Kotani, and V. R. Algazi, "Objective picture quality scale (PQS) for image coding," IEEE Trans. Commun., vol. 46, no. 9, pp. 1215-1226, Sep. 1998.

[4] Z. Wang, E. P. Simoncelli, and A. C. Bovik, "Multi-scale structural similarity for image quality assessment," in Proc. IEEE Asilomar Conf. Signals, Syst., Comput., Nov. 2003, pp. 1398-1402.

[5] S. Rezazadeh and S. Coulombe, "A novel approach for computing and pooling structural similarity index in the discrete wavelet domain," in Proc. Int. Conf. Image Process., Cairo, Egypt, Nov. 2009, pp. 22092212.

[6] D. M. Chandler and S. S. Hemami, "VSNR: A wavelet-based visual signal-to-noise ratio for natural images," IEEE Trans. Image Process., vol. 16, no. 9, pp. 2284-2298, Sep. 2007.

[7] H. R. Sheikh and A. C. Bovik, "Image information and visual quality," IEEE Trans. Image Process., vol. 15, no. 2, pp. 430-444, Feb. 2006.

[8] H. R. Sheik, M. F. Sabir, and A. C. Bovik, "A statistical evaluation of recent full reference image quality assessment algorithms," IEEE Trans. Image Process., vol. 5, no. 11, pp. 3441-3452, Nov. 2006.

[9] K. Seshadrinathan and A. C. Bovik, "Unifying analysis of full reference image quality assessment," in Proc. IEEE Int. Conf. Imge Process., Oct. 2008 , pp. $1200-1203$

[10] A. Horé and D. Ziou, "Image quality metrics: PSNR vs. SSIM," in Proc. IEEE Int. Conf. Pattern Recognit., Istanbul, Turkey, Aug. 2010, pp. 2366-2369.

[11] C. Charrier, L. T. Maloney, H. Cherifi, and K. Knoblauch, "Maximum likelihood difference scaling of image quality in compression-degraded images," J. Opt. Soc. Amer. A, vol. 24, no. 11, pp. 3418-3426, 2007.

[12] L. T. Maloney and J. N. Yang, "Maximum likelihood difference scaling," J. Vis., vol. 3, no. 8, pp. 573-585, 2003.

[13] K. Knoblauch and L. T. Maloney, "MLDS: Maximum likelihood difference scaling in R," J. Stat. Softw., vol. 25, no. 2, pp. 1-26, 2008.

[14] K. Knoblauch and L. T. Maloney, Modeling Psychophysical Data in R. New York: Springer-Verlag, 2012.

[15] S. Stevens, "On the theory of scales measurement," Science, vol. 103, no. 2684 , pp. $677-680,1946$.

[16] D. H. Kra, "A theory of magnitude estimation and cross-modality matchings," J. Math. Psychol., vol. 9, no. 2, pp. 168-199, 1972.

[17] R. N. Shepard, "Psychological relations and psychophysical scales: On the status of direct psychophysical measurement," J. Math. Psychol., vol. 24 , no. 1 , pp. 21-57, 1981.

[18] D. M. Green and J. A. Swets, Signal Detection Theory and Psychophysics. Malabar, FL: Robert E. Krieger Publishing, 1974

[19] Z. Wang, A. C. Bovik, H. R. Sheikh, and E. P. Simoncelli, "Image quality assessment: From error visibility to structural similarity," IEEE Trans. Image Process., vol. 13, no. 4, pp. 600-612, Apr. 2004.

[20] The JasPer Projet. (1999). Univ. Victoria, Victoria, BC, Canada [Online]. Available: http://www.ece.uvic.ca/ mdadams/jasper/

[21] D. M. Rouse and S. S. Hemami, "Understanding and simplifying the structural similarity metric," in Proc. Int. Conf. Image Process., 2008, pp. 1188-1191.

[22] C. Charrier, K. Knoblauch, A. K. Moorthy, A. C. Bovik, and L. T. Maloney, "Comparison of image quality assessment algorithms on compressed images," Proc. SPIE, Image Qual. Syst. Perform. VII, vol. 7529, pp. 75290B-1-75290B-11, Jan. 2010.

[23] M. Srinivas and L. M. Patnaik, "Genetic algorithms: A survey," IEEE Comput., vol. 7, no. 6, pp. 17-26, Jun. 1994.

[24] J. H. Holland, Adaptation in Natural and Artificial Systems. Cambridge, MA: MIT Press, 1992

[25] K. D. Jong, An Analysis of the Behavior of a Class of Genetic Adaptive Systems. Ann Arbor, MI: Univ. Michigan Press, 1975.

[26] D. Goldberg, Genetic Algorithms in Search, Optimization and Machine Learning. Reading, MA: Addison-Wesley, 1989.

[27] Y. Wang and K. Fan, "Recognition of occluded objects by a genetic algorithm," in Proc. IEEE Int. Conf. Pattern Recognit., Vienna, Austria, Aug. 1996, pp. 740-744.

[28] L. Zhang, W. Xu, and C. Chang, "Genetic algorithm for affine point pattern matching," Pattern Recognit. Lett., vol. 24, nos. 1-3, pp. 9-19, 2003.

[29] M. Benedetti, M. Donelli, and A. Massa, "Multicrack detection in twodimensional structures by means of GA-based strategies," IEEE Trans. Antennas Propag., vol. 55, no. 1, pp. 205-215, Jan. 2007.

[30] Final Report from the Video Quality Experts Group on the Validation of Objective Models of Video Quality Assessment. (2000) [Online]. Available: https://www.itu.int/md/T01-SG09-C-0060/en

[31] G. W. Snedecor and W. G. Cochran, Statistical Methods, 8th ed. New York: Wiley, 1990.

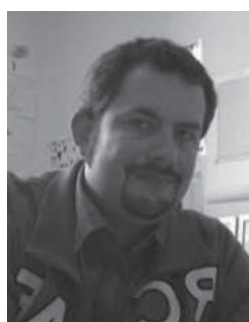

Christophe Charrier (M'10) received the M.S. degree from the Nantes University of Science and Technology, Nantes, France, in 1993, and the Ph.D. degree from the Université Jean Monnet, SaintÉtienne, France, in 1998

$\mathrm{He}$ is with the University of Caen BasseNormandie, Caen, France, since 2001, where he is a member of the Image Team, GREYC Laboratory. $\mathrm{He}$ is a Faculty Member with the Communication, Networks and Services Department, Cherbourg Institute of Technology, Cherbourg, France. His research activities are currently performed with the GREYC laboratory, University of Caen Basse-Normandie. From 1998 to 2001, he was a Research Assistant with the Laboratory of Radio Communications and Signal Processing, Laval University, Quebec, QC, Canada. From 2003 to 2006, he was the Director of the Services and Network Communication Department, University of Caen Basse-Normandie, France. In 2008, he was a Visiting Scholar with the Laboratory for Image and Video Engineering, University of Texas at Austin, Austin. From 2009 to 2011, he was an Invited Professor with the Computer Department, University of Sherbrooke, Sherbrooke, QC, Canada. His current research interests include digital image and video coding, processing, quality assessment, and computational vision.

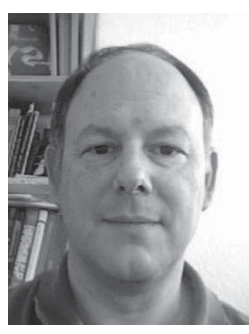

Kenneth Knoblauch is a Researcher with INSERM Unit 846, Department of Integrative Neurosciences, Institut National de la Santé et de la Recherche Médicale, Stem Cell and Brain Research Institute, Bron, France, and the University Claude Bernard Lyon 1, Lyon, France. Prior to joining INSERM, he was an Associate Professor with the Institute of Vision Engineering, University Jean Monnet of Saint Etienne, France, in 2005. Before moving to France, he was a Senior Investigator for seven years with the Research Department, Lighthouse, Inc., New York, NY. He has been involved in research on low vision, functional cerebral imagery, and development and aging of visual function. He is the co-author of the forthcoming Modeling Psychophysical Data in R. His current research interests include perception and sensory coding, color vision, psychophysics, and modeling of neural processes.

$\mathrm{He}$ is a member of the Board of the Directors of the International Colour Vision Society and is on the Editorial Board of the Journal of Statistical Software. In 2009, he was invited to give the David Palmer Lecture to the Colour Group of Great Britain.

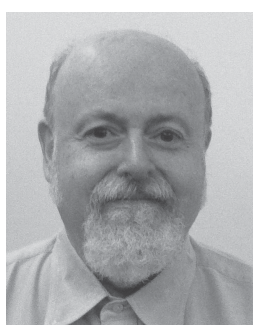

Laurence T. Maloney received the B.A. degree in mathematics from Yale University, New Haven, CT, in 1973, and the M.S. degree in mathematical statistics and the Ph.D. degree in psychology from Stanford University, Stanford, CA, in 1982 and 1985 , respectively.

$\mathrm{He}$ is currently a Professor of psychology and neural science with New York University, New York, NY. He was a Resident with the Center for Interdisciplinary Studies, Bielefeld University, Deutschland, Germany, from 1995 to 1996 , as part of a group considering perception and evolution. In 1998, he was the Forchheimer Visiting Professor with Hebrew University, Jerusalem, Israel, and in 2009, the WICN Scholar with Bangor University, Wales, U.K. He has been a Visiting Professor at several other universities, including the University of Freiburg, Baden-Württemberg, Germany, the University of Giessen, Hesse, Germany, the University of Paris, Paris, France, and the University of Padova, Padua, Italy. His research interests include applications of mathematical models to understanding human behavior. His work in the physics and mathematics of color vision resulted in two articles in the Journal of the Optical Society of America, which are frequently cited in published works. His previous research on visual cue combination led to a frequently cited review article in Vision Research. His recent research on movement planning and decision making under risk has built a bridge between two unrelated fields. He has authored or co-authored more than 140 published articles, proceeding papers, and chapters, and co-authored the forthcoming Modeling Psychophysical Data in $R$.

Dr. Maloney was a recipient of the Humboldt Research Award from the Humboldt Foundation of Germany in 2008, and the Troland Award from the National Academy of Sciences in 1987. He is a fellow of the Association for Psychological Science and of the Society of Experimental Psychologists. He was a recipient of the One-Year Research Fellowship from the Center for Interdisciplinary Studies at Bielefeld University through the German Research Council in 1994. 


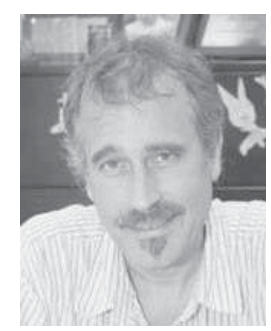

Alan C. Bovik (F'96) is the Curry/Cullen Trust Endowed Chair Professor with the University of Texas at Austin, Austin, where he is the Director of the Laboratory for Image and Video Engineering. He is a Faculty Member with the Department of Electrical and Computer Engineering, Center for Perceptual Systems, Institute for Neuroscience. He is a frequent consultant to legal, industrial, and academic institutions. He has authored or co-authored more than 600 published technical articles and holds two U.S. patents. His several books include the recent companion volumes, The Essential Guides to Image and Video Processing (Academic Press, 2009). His current research interests include image and video processing, computational vision, and visual perception.

He was a recipient of two Journal Paper Awards from the International Pattern Recognition Society in 1988 and 1993, the IEEE Third Millennium Medal in 2000, the Hocott Award for Distinguished Engineering Research, University of Texas, in 2008, the Distinguished Alumni Award from the University of Illinois at Urbana-Champaign, Urbana, in 2008, the SPIE/IS\&T Imaging Scientist of the Year in 2011, as well as a number of major awards from the IEEE Signal Processing Society, including the Meritorious Service Award in 1998, the Technical Achievement Award in 2005, the Education Award in 2007, and the Best Paper Award in 2009. He is a fellow of the Optical Society of America, the Society of Photo-Optical and Instrumentation Engineers, and the American Institute of Medical and Biomedical Engineering. He has been involved in numerous professional society activities, including the Board of Governors of the IEEE Signal Processing Society from 1996 to 1998 . He is a Professional Engineer of the State of Texas.
He is the co-founder and was the Editor-in-Chief of the IEEE TRANSACTIONS ON IMAGE PROCESSING from 1996 to 2002. He was on the Editorial Boards of the PROCEEDINGS OF THE IEEE from 1998 to 2004, a series editor for Image, Video, and Multimedia Processing (Morgan and Claypool Publishing Company, 2003), and the Founding General Chairman of the first IEEE International Conference on Image Processing, Austin, TX, in 1994.

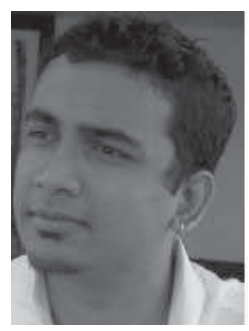

Anush K. Moorthy received the B.E. degree (silver medal) in electronics and telecommunication from the University of Pune, Pune, India, in 2007, and the M.S. degree in electrical engineering from the University of Texas at Austin, Austin, in 2009, where he is currently pursuing the $\mathrm{Ph} . \mathrm{D}$. degree in electrical and computer science.

$\mathrm{He}$ joined the Laboratory for Image and Video Engineering (LIVE), University of Texas at Austin, in 2008, and is currently an Assistant Director with the Department of Electrical and Computer Engineering, LIVE, University of Texas. His current research interests include image and video quality assessment, image and video compression, and computational vision.

Mr. Moorthy was a recipient of the Continuing Graduate Fellowship from the University of Texas at Austin for 2010 to 2011, the Professional Development Award in 2009 and 2010, the Center for Perceptual Systems Travel Grant in 2010, and the TATA Scholarship for Higher Education Abroad. 